\title{
QUAND L'ÉCOLOGIE DEVIENT NUISANCE
}

\author{
J. GADANT
}

Après celle de Stockholm, la $2^{\mathrm{e}}$ Conférence des Nations-Unies sur l'Environnement et le Développement (CNUED), dite "Sommet de la terre", se tenait à Rio de Janeiro en juin 1992. Elle a rassemblé 40000 participants, 115 chefs d'État et de Gouvernement.

L'intérêt transfrontalier et universel de la forêt, dans tous les pays du monde, du fait de la pollution atmosphérique, du maintien des climats, de la sauvegarde de la biodiversité, explique le succès de ce grand rassemblement international. II résulte aussi de la régression des forêts tropicales, de leur dégradation en zones arides qui suscitent de légitimes inquiétudes et un déluge de débats internationaux.

Bien sûr, les forestiers, méconnus durant des siècles, se réjouissent aujourd'hui de cette brusque et salutaire prise de conscience qui a tant tardé. Faut-il encore que le tintamarre actuel n'étouffe pas l'expression des vrais problèmes et que les voix compétentes puissent se faire entendre.

Tel n'est pas toujours le cas. L'inflation de débats et de rapports s'alimentant de vieilles et coriaces fausses idées génère une extrême confusion qui retarde la mise en œuvre des vraies solutions. II faut dire que certaines Organisations non gouvernementales (ONG) répandent le catastrophisme forestier planétaire, orchestrent de mauvaises querelles, préfèrent la critique facile des responsables de la gestion forestière, plutôt que de proposer des solutions réalisables et d'y participer concrètement.

\section{LES DÉTESTABLES FAUX DÉBATS}

En 1995, la revue Unasylva de la FAO avait bien voulu ouvrir ses colonnes au "point de vue" de l'Association "Greenpeace". Sous le titre: "Utilisation écologiquement responsable des forêts" et sous la signature de son directeur international $P$. Hohnen, la première phrase donne immédiatement le ton : "Une chose est claire: les forêts qui restent dans le monde sont en crise et ne survivront pas 50 ans de plus si on leur applique les mêmes pratiques qui ont marqué le demi-siècle passé ".

Ce constat global alarmiste, qui loge tous les pays du monde à la même enseigne de la destruction, qui ne nuance pas des situations géographiques extrêmement diverses, est totalement faux, consti- 
tue un bel exemple d'ignorance ou de tromperie. En France, par exemple, étant donné les politiques et les actions qui sont menées, il n'y a aucune raison pour que nos patrimoines forestiers, assujettis depuis longtemps à des rendements soutenus, ne soient pas, dans un siècle, dans l'état où ils étaient au siècle précédent. S'il est vrai que des pays ont inconsidérément surexploité leurs forêts sans les reconstituer, il faut tout de même savoir que l'Europe et de nombreux pays à travers le monde ont mené d'actives politiques de reboisement et que leur avenir forestier est prometteur si les réalisations du passé peuvent être poursuivies.

La crise évoquée par M. Hohnen concerne essentiellement les pays tropicaux en développement où règne la pauvreté et où les forêts sont pratiquement dans un état d'absence d'une gestion conservatrice du capital ligneux. Il y a belle lurette que les forestiers en ont fait le constat, notamment l'organisme habilité et compétent du système des Nations-Unies qu'est la FAO. Son concept de plan d'action forestier tropical (PAFT) vise précisément à promouvoir des politiques de gestion forestière adaptées à chaque pays.

Le débat international est utile dans la mesure où il peut mobiliser les opinions, peser sur les responsables politiques des États. Mais il est nécessairement trop général et théorique pour être capable de préconiser des solutions concrètes et opérationnelles. Par ailleurs, un discours trop globalisé et mondialisé sur la forêt est trompeur : ce qui est vrai ici est souvent faux ailleurs. À des situations socio-écologiques très changeantes répondent des diagnostics nécessairement nuancés et des solutions adaptées. On ne traite pas la forêt méditerranéenne comme la forêt vosgienne; la forêt canadienne est un cas, la forêt sahélienne en est un autre. Pour être vrai et court, au risque d'être provoquant, disons que la forêt mondiale, au singulier, n'existe pas!

Ainsi, en France, où la géologie est variée et les climats multiples, notre plan forestier national est modulé localement par des orientations et des directives forestières régionales, elles-mêmes adaptées concrètement dans les plans d'aménagement ou de gestion qui sont mis en œuvre par des sylviculteurs au niveaus de chaque forêt.

Par ailleurs, hors la prédiction apocalyptique, le coupable du prétendu saccage de la forêt mondiale est désigné : "'exploitation du bois". Bien sûr, il y a des abus et c'est précisément pour les éviter que des méthodes de gestion à "rendement soutenu" ont été imaginées dans un passé déjà lointain, ainsi qu'une panoplie bien fournie de réglementations protectrices limitant les droits des propriétaires. Mais, sur ce point, certains écologistes internationaux commettent une autre grave erreur en s'acharnant à minimiser le rôle économique de la forêt jusqu'à exiger "que le rôle des industries forestières dans la dégradation des forêts soit reconnu". La coupe abusive existe et doit être proscrite ; mais les véritables causes de la régression des forêts tropicales ont été maintes fois analysées et sont aujourd'hui bien connues: la pauvreté rurale, la surexploitation du bois de feu par manque d'énergie, le surpâturage, le besoin en terre pour nourrir des populations qui ont faim, etc.

Mais la cause essentielle est l'absence d'une véritable gestion car on ne peut pas raisonnablement contester :

- d'une part, qu'une exploitation rationnelle de la forêt, écologiquement attentive, est un acte de gestion indispensable si l'on souhaite que la forêt puisse remplir l'ensemble de ses fonctions économique, écologique et sociale. Le vrai problème est de bien planifier, sur de longues périodes, la quotité et la localisation de ces coupes sauvegardant les intérêts protecteurs et écologiques de la forêt ;

- d'autre part, que le bois est le seul revenu qui couvre l'ensemble du coût de gestion supporté par un propriétaire qui investit, prend des risques, assume des dépenses. Le devoir des pouvoirs publics pour satisfaire les exigences de la société est d'encourager et d'aider ce propriétaire qui réalise une œuvre d'intérêt général. Tous les bavardages irresponsables sur la forêt auraient plutôt tendance aujourd'hui à démobiliser cette précieuse "poule aux œufs d'or". 


\section{LES IDÉES REÇUES}

Sur un sujet aussi complexe et divers, toujours imprégné de passion, où il suffit d'être intéressé pour devenir compétent, où s'imposent la prudence et la nuance, il est inévitable que de généreux théoriciens en arrivent à formuler des idées erronées d'autant plus dangereuses qu'elles peuvent être séduisantes. Leurs affirmations, amplifiées par les médias, polluent le débat forestier.

Le scientifique forestier allemand Thomas Bouman a procédé à une analyse des propositions de Knut Sturm de "Greenpeace Deutschland"; il conclut que l'Organisation écologique "n'a pas compris le concept d'une gestion forestière axée sur plusieurs objectifs et, a fortiori, dans des contextes culturels différents ", s'en tenant à un intégrisme écologique excessif, de pure conservation.

À titre d'exemple, pour montrer l'intérêt d'une gestion plurifonctionnelle de la forêt, citons les ambitieux reboisements de la restauration des terrains en montagne réalisés au siècle dernier, dans un objectif principal essentiellement protecteur. Il s'agissait de lutter contre les érosions et les inondations et non pas de produire du bois. II se trouve aujourd'hui, alors que l'objectif initial (écologique) est réalisé, que des coupes doivent y être pratiquées (aspect économique), que ces montagnes sont parées d'un beau manteau boisé (aspect paysager), tandis que de merveilleux sentiers balisés s'ouvrent aux promeneurs (aspect social). Comment faire mieux?

La gestion à usages multiples est la seule réponse à la diversité de la demande de notre société. L'erreur est de se montrer, soit trop productiviste, soit trop conservateur. Sur le plan de la conservation, le réseau européen "Natura 2000 " se met en place ; il est explicité dans une bonne directive européenne. Une mise en œuvre déraisonnable pourrait polluer la conception initiale. Le premier projet proposait le classement de 8 millions d'hectares en zones spéciales de conservation, soit $15 \%$ du territoire national. Élaboré par des intégristes de l'écologie, sans prise en compte de critères économiques et sans concertation, il a été dénoncé ("cri d'alarme" du 10 avril 1996) par 9 grandes organisations professionnelles, agricoles et forestières, et heureusement atténué. Par ailleurs, il est bien évident qu'une gestion rationnelle des sites forestiers retenus exigera que les mesures de conservation soient intégrées dans les documents d'aménagement et mises en œuvre par les gestionnaires de la forêt.

La hache de guerre n'a pas encore été enterrée dans le combat inutile "feuillus-résineux". Le philosophe Bertrand de Jouvenel, qui avait été chargé par le Gouvernement d'une réflexion sur la forêt, m'avait confessé son erreur, puis écrit: "J'étais hostile aux résineux, mais c'est affaire de lieux". Allez dire aux Vosgiens ou aux Landais que leurs sapins et leurs pins sont tristes et laids et que des feuillus seraient plus esthétiques.

L'opposition aux essences dites "exotiques" relève d'un racisme végétal sans fondement scientifique. De nombreuses introductions se sont révélées bénéfiques au plan sylvicole, bien intégrées et naturellement conquérantes. Le Pin noir d'Autriche, le Cèdre de l'Atlas nord-africain, le Robinier de l'Est américain, le Douglas de l'Ouest américain et bien d'autres venus d'ailleurs font, sous nos climats, de très brillantes carrières.

La Conférence de Rio a donné la définition suivante de la diversité biologique : "Variabilité des organismes vivants, de toutes origines, y compris les écosystèmes terrestres, marins et aquatiques. Cela comprend la diversité au sein des espèces, entre espèces, ainsi que celle des écosystèmes". Au plan forestier, les inquiétudes relatives à la biodiversité sont inspirées de la dramatique régression des forêts tropicales dont la richesse floristique exceptionnelle est menacée. En zone aride, bien audelà d'un simple appauvrissement, la désertification élimine des espaces boisés. Partout la conservation des espèces appelle une attention particulière, mais les cris d'alarme sont largement exagérés dans nos forêts d'Europe.

Rien ne peut justifier, au plan scientifique, une interdiction généralisée de la coupe rase d'un peuplement parvenu à maturité ; elle existe dans la nature (incendie, chablis, maladie) et répond à une 


\section{J. GADANT}

nécessité culturale dans un traitement en taillis ou une régénération d'essences de pleine lumière. Les forestiers finlandais réputés protecteurs récoltent la moitié de leurs bois par coupes rases.

On a aussi imaginé des normes auxquelles la gestion forestière devrait se soumettre, par exemple, sur la densité des chemins, les pourcentages de bois à prélever et de la biomasse à conserver. Elles sont souvent irréalistes et dangereuses. À ce point de vue encore, ce qui est vrai ici est erroné ailleurs ; laissons faire le coup d'œil compétent du praticien de la sylviculture.

Enfin, au niveau international, des organisations écologiques au chevet de la forêt ont essentiellement en tête les forêts de l'État, très dominantes dans les pays en développement. Ils oublient la forêt privée, les problèmes particuliers qu'elle pose, l'intérêt qu'elle présente, notamment en responsabilisant les nombreux propriétaires qui la possèdent.

Hors quelques entreprises de ligniculture qui s'apparentent à une production agricole, la gestion forestière demeure très extensive; elle a peu recours aux fertilisants, les traitements sanitaires et phytocides y sont rares. II est déraisonnable de s'opposer, par principe, à la fertilisation d'une plantation ou à un dégagement chimique qui interviennent à la périodicité du siècle, alors que la production agricole les multiplie chaque année.

L'accent est mis aujourd'hui sur l'écosystème forestier, la diversité des espèces animales et végètales qui le constituent el la complexité de leurs interactions. Une espèce composante de cet écosystème est parfois oubliée: c'est l'homme. Rien ne peut justifier que ce dernier ne cherche pas à en tirer profit. En clair, s'agissant des pays tropicaux et de leur grande pauvreté, peut-on imposer la création de réserves forestières intégrales ou s'opposer à des défrichements à but agricole, ou y proscrire le pâturage, quand les malheureuses populations du voisinage manquent de terres à cultiver pour apaiser leur faim?

\section{LES CONTRAINTES OUBLIÉES}

Mais il n'y a pas que les tausses idées qui polluent les débats forestiers; il y a aussi les vraies contraintes qui sont mal connues ou mal comprises: le très long terme, un revenu modeste, aléatoire et longtemps différé, l'absence de volonté politique des États, l'insuffisance des moyens affectés, l'ignorance de la vraie valeur des services immatériels que la forêt rend à nos sociétés, etc., etc. Les "il n'y a qu'à" ou "il faut qu'on" sont faciles ; la question "qui paie ?" est plus rarement posée. Elle est pourtant essentielle : en effet, si on conserve un écosystème en le classant, c'est que la biodiversité qu'il recèle a une valeur à laquelle il faudra bien attribuer un prix.

La fonction de production de bois est le socle sur lequel la gestion forestière peut asseoir une capacitè de rendre des services d'intérêt gẻnéral aux plans écologique et social. L'objectif de production de bois n'a rien de mercantile car il faut savoir aussi que la rentabilité forestière est faible et assujettie à des risques. Par ailleurs, ces propriétaires ne poursuivent pas tous un objectif économique de production de bois; la réalité est beaucoup plus complexe et nuancée, leurs motivations variées et souvent confuses: l'aménagement d'un paysage, l'agrément de contempler des arbres qui changent avec les saisons, le plaisir d'enrichir et d'élever un peuplement, chargẻ d'affectivité et d'histoire, reçu des anciens et à transmettre aux jeunes. $\dot{A}$ chacun sa forêf. Et, en conséquence, à chacun sa gestion.

Nos sociétés assignent aujourd'hui aux forêts des fonctions de plus en plus diversifiées; ces nouvelles exigences compliquent singulièrement la tâche des gestionnaires, enlevant toute signification aux théories simplistes des débats planétaires. Il n'y a jamais une forêt à faire seulement du bois, comme il y a une betterave à faire du sucre. Pour le citadin enfermé dans les nuisances de sa ville, la forêt est un havre de nature ; ailleurs, elle ouvre aux promeneurs ses sentiers, ses espaces silen- 
cieux de méditation; ailleurs, elle est un refuge de la faune sauvage pour celui qui la chasse ou celui qui l'observe ; ailleurs, elle est un écosystème complexe, encore mal connu, et le réservoir d'une précieuse biodiversité à sauvegarder; ailleurs, elle est une merveilleuse fabrique d'oxygène, un filtre épurateur de la pollution et un étonnant piège de ce gaz carbonique dont la nocivité est tant redoutée aujourd'hui ; partout, elle secrète du bois, écomatériau renouvelable, générant de l'activité économique et des emplois. Bien sûr, toutes ces exigences imposent des arbitrages, mais le rôle du forestier gestionnaire est précisément de limiter ces "ailleurs" en s'efforçant de pratiquer une gestion plurifonctionnelle et de faire cohabiter le maximum de ces fonctions sur le même espace.

Durant le Congrès forestier mondial tenu à Paris en 1991, nous avons pu conduire près de 2000 participants de 140 pays du monde à travers les futaies de la forêt domaniale de Bellême. Tous ont manifesté leur étonnement et leur admiration pour ce vieux monument végétal, constamment régénéré, parfaitement conservé, soumis à une gestion planifiée, plurifonctionnelle, durable.

\section{LA MAUVAISE COMPRÉHENSION DE LA FORÊT ET DU BOIS}

Ainsi les vrais problèmes de la forêt demeurent encore mal compris. Notre patrimoine forestier est mal connu dans son importance, sa composition, la variété de ses fonctions, le rôle des gestionnaires, la diversité des opérateurs qui composent l'ensemble de la filière de la forêt et du bois.

Quelques statistiques incontestées chiffrent son importance en France : $27 \%$ de taux de boisement, un doublement de la superficie boisée en 2 siècles, une production biologique qui approche 80 millions de mètres cubes, une récolte de l'ordre de 50 millions de mètres cubes laissant le patrimoine sous-exploité, une filière créatrice de 600000 emplois, etc.

Voilà de quoi rayonner d'optimisme. Mais qu'en disent les médias ? Ils ignorent la prospérité silencieuse de la forêt; ils en parlent quand elle brûle, quand des tempêtes la saccagent, quand des pollutions l'affectent, quand des maladies l'agressent. Et qu'en pensent les Français ? Une enquête d'opinion de 1992 donne des chiffres surprenants:

$-85 \%$ pensent qu'elle est gravement menacée alors qu'elle continue de s'étendre et de s'enrichir,

— Cette idée fausse d'une forêt qui régresse et se dégrade conduit $87 \%$ d'entre eux à souhaiter que notre patrimoine forestier soit affecté à des réserves naturelles, utilisé comme lieu de détente, etc.

$-13 \%$ seulement pensent à l'intérêt économique, donc social, d'y exploiter du bois. Et pourtant, les spécialistes ont foujours affirmé que les diverses fonctions que la forêt doit satisfaire sont parfaitement compatibles et que son exploitation régulière était indispensable pour en assurer la pérennité et conserver son aspect accueillant.

En présentant à la presse, le 7 février 1996, la politique forestière qu'il entend mener, le Ministre Vasseur a mis l'accent sur cet objectif économique, d'autant plus que nos ressources en bois vont en augmentant : améliorer la compétitivité de la filière, rechercher de nouveaux débouchés, exporter davantage. Voilà qui est clair! Le Président Chirac a proclamé que tout secteur d'activité devait contribuer à la réduction du chômage. Va-t-on en exclure le quart du territoire national ?

Par ailleurs (et cela est pour moi un mystère), le message vrai du bois "écomatériau", à la fois esfhétique, chaleureux, facile à travailler, aux usages si divers, n'arrive pas à passer. II est pourtant simple :

- le bois est renouvelable et facilement recyclable;

- sa mise en œuvre réalise une énorme économie d'énergie comparée aux matériaux qui le concurrencent (béton, métaux, verre, plastiques); 
- contrairement à une idèe reçue, il résiste bien à la pourriture et au feu quand il a été convenablement séché, traité et correctement mis en œuvre comme l'attestent ces élégantes charpentes conservées en de vieux bâtiments ;

- enfin, il réalise un étonnant stockage de gaz carbonique, compensant ce dangereux "effet de serre" tant redouté de l'écologie planétaire.

La forêt française a ses problèmes qu'il convient de traiter; mais elle a aussi ses succès que des chiffres éloquents proclament. Cette expansion et cette prospérité sont le fruit de gros efforts de gestion que le seul qualificatif de "durable" minimise puisqu'elle s'accroît et s'enrichit. Et pourtant l'incompréhension demeure.

Ce surprenant divorce entre l'opinion de certains Français et cette réalité forestière peut inspirer un premier constat: la forêt et le bois souffrent d'un déficit de communication considérable. On peut accuser les médias trop friands de catastrophisme; on peut reprocher aux forestiers qui savent gérer de ne pas savoir expliquer la forêt! Mais il y a aussi ceux qui refusent d'entendre, préférant se valoriser dans la contestation et l'accusation! Ils jouent un rôle néfaste de désinformation de l'opinion publique. Cette écologie dévoyée en arrivera-t-elle à devenir une nuisance pour la forêt ?

\section{LES GESTIONNAIRES ACCUSÉS}

Ainsi, au plan international, comble de l'incompréhension, voilà que les gestionnaires des forêts sont accusés de mal gérer. En présentant un numéro de 1995 d'Unasylva, le sous-directeur général de la FAO, responsable du Département des Forêts, en fait le constat: "Les forestiers risquent d'être écartés des décisions dans les domaines dont ils s'occupent".

Sans doute, le reproche peut être fait aux forestiers d'avoir négligé d'expliquer davantage. Mais le dialogue est souvent difficile tant il est vrai qu'il y a, d'un côté ceux qui ont la rude responsabilité de taire en négligeant peut-être de dire et, de l'autre, ceux qui abusent de la facilité de dire sans se soucier de faire.

Ainsi, l'incroyable reproche serait fait aux forestiers, orgueilleusement accrochés à leurs certitudes, d'être enchaînés à leur savoir et leurs techniques! Le sont-ils plus que le médecin dans sa médecine, le pilote d'avion en face de ses cadrans et tout spécialiste qui exerce un métier qu'il a appris et qui ne s'improvise pas ? La sylviculture et l'écologie sont des disciplines qui s'apprennent, mais aussi un art qui se cultive et une longue expérience dont on se dote avec le temps.

J'ai effectué de longues études pour accéder à un parchemin public me reconnaissant du savoir en écologie et de l'aptitude à la sylviculture. Après 40 années, j'en suis arrivé inévitablement à quelques prétentions sur ce sujet. C'est pourquoi, confronté à des critiques et des erreurs tenaces, j'en suis arrivé aussi à quelques irritations.

En effet, jamais il ne me serait venu à l'idée de prétendre à une compétence militaire devant un génèral ou d'enseigner la philosophie à un philosophe. Curieusement l'inverse n'est pas vrai et on peut entendre ici un général, là un botaniste ou un sociologue, proclamer la vérité forestière, sans toujours l'appuyer sur une formation ou une expérience.

La FAO m'avait convié, en mars 1995, à une réunion de concertation avec des ONG internationales. La réunion, dominée par les grandes organisations internationales dites "écologiques", fut constamment polémique et décevante : contestation systématique des actions menées par la FAO, critiques insensées des gestionnaires des forêts. J'étais intervenu sur quatre points: les difficultés d'une appréhension mondialisée des problèmes forestiers, la gestion plurifonctionnelle des forêts, un avenir d'écomatériau pour le bois, le rôle irremplaçable des propriètaires-sylviculteurs. J'eus pour réponse un silencieux mépris. 
Ayant refusé de m'associer aux conclusions proposées, dans un article publié par "Silva", j'avais relaté ainsi les conclusions que m'inspirait cette étrange rencontre:

- "Le dangereux abandon du débat international sur la forêt à des théoriciens intégristes de l'environnement, parfois même à de séduisantes incompétences".

- "Une impression de tourner en rond dans la manipulation de concepts fumeux, sans les transformer en actions concrètement réalisables ".

- "L'utopie d'une politique forestière mondialisée qui ne peut déboucher que sur des recommandations et de longs catalogues de mesures".

Après avoir longtemps recherché un accord sur un "Instrument international sur les forêts", les débats de la Conférence de Rio ont débouché sur une "Déclaration de principes non juridiquement contraignante, mais faisant autorité, en vue d'un consensus mondial sur une gestion écologiquement fiable de tous les types de forêt $"$. Ce texte artistement ciselé révèle les difficultés du débat international et ne constitue qu'une généreuse déclaration d'intention. Mais on eut cependant la grande sagesse de ne pas prétendre à un texte mondialisé de politique forestière et de limiter l'ambition à ce code de bonne conduite que les États s'engagent à mettre en œuvre.

\section{LA GESTION FORESTIÈRE}

Les conclusions de la Conférence de Rio contiennent un dénominateur commun important, de portée universelle, applicable à tous les pays et à toutes les forêts: c'est l'exigence d'une bonne gestion qui soit valorisatrice de tous les biens et services que rend la forêt, conservatrice du patrimoine, protectrice de la diversité biologique. On la nomme aujourd'hui "gestion durable"; elle était dite hier "gestion à rendement soutenu".

II ne faudrait pas croire que cette préoccupation est nouvelle dans les pays de vieille tradition forestière. Dans sa lettre de bons vœux pour l'année 1996, l'Office national des Forêts nous rappelle que l'Ordonnance de Brunoy de Philippe VI de Valois définissait déjà, en 1346, le principe d'une gestion forestière durable: "Les Maitres des Forêts enquerront et visiteront toutes les forêts et bois qui y seront et feront les ventes qui y sont à faire, eu regard à ce que les dites forêts se puissent perpétuellement soutenir en bon état ". La lettre nous rappelle aussi que le patrimoine forestier d'aujourd'hui a retrouvé l'étendue de celui que Philippe VI a connu : est-il possible d'être plus durable!

Thomas Bouman, scientifique forestier d'Allemagne, est plus modeste en rappelant "que le désir de préserver à long terme les ressources forestières est né en Allemagne il y a 400 ans, à une époque où une pénurie de bois commençait de se faire sentir".

\section{L'EXPÉRIENCE D'HIER : LA GESTION À RENDEMENT SOUTENU}

Elle est mise en œuvre depuis longtemps dans le cadre de plan d'aménagement et de gestion et de règlement d'exploitation. L'objectif est d'assurer la conservation du capital ligneux, de veiller à un bon équilibre des classes d'âge, de prélever le volume de l'accroissement, d'améliorer et de régénérer les peuplements, etc. Deux exemples simplistes:

— L'aménagement (en surface) d'un taillis simple de 100 hectares, à une révolution de 25 ans, exploite chaque année 4 hectares et assure le rendement soutenu et la pérennité de la forêt.

- L'aménagement (en volume) d'une futaie de 100 hectares, dont l'accroissement annuel est de 6 mètres cubes, prélève $600 \mathrm{~m}^{3}$ par an, laissant la forêt inchangée.

Cette notion de rendement soutenu a l'avantage de mettre l'accent sur le bon équilibre des exploitations à réaliser, tant il est vrai qu'il y a deux façons de mal gérer : soit surexploiter et appauvrir le 


\section{J. GADANT}

capital ligneux, soit sous-exploiter et acheminer les peuplements vers le dépérissement, une perte de croissance et des difficultés de régénération naturelle. II est tout de même cocasse que ceux qui dénoncent aujourd'hui l'insuffisance de cette gestion à rendement soutenu sont précisément ceux qui considèrent que les exploitations mal maîtrisèes et excessives sont la cause essentielle de la destruction des forêts tropicales.

Hors la conservation des peuplements, le rendement soutenu présente aussi l'avantage de réguler les exploitations de bois; ainsi au plan de l'emploi et de l'activité économique, il contribue à garantir un travail régulier à la main-d'œuvre d'exploitation et un approvisionnement régulier des industries du bois.

Enfin, le seul fait d'assurer la pèrennité du capital ligneux peut contribuer à sauvegarder la possibilité d'exercice des autres fonctions. D'ailleurs, ces autres fonctions n'ont jamais été étrangères à ces aménagements. Il y a longtemps qu'un objectif d'accueil du public a été assigné à certaines forêts d'lle-de-France et d'ailleurs, à proximité des villes. Dans ce cadre, des réserves biologiques ou artistiques, des séries cynégétiques, ont été créées. Au siècle dernier, pilotés par des aménagements de défense et de restauration des sols en montagne, les reboiseurs de l'Aigoual ne poursuivaient pas un objectif de production, mais luttaient essentiellement contre les érosions et les inondations, etc., etc.

Bien sûr, il y a toujours des insuffisances et des erreurs. Peut-être, ici et là, a-t-on trop uniformisé les peuplements, minimisé l'esthétique et le paysager, porté insuffisante attention à la protection d'espèces menacées. Sans doute, l'appellation "rendement soutenu" présente le travers de faire apparemment la part trop belle aux ligneux. La forêt est bien autre chose que du bois en croissance ; elle est aussi une histoire, une ambiance, une culture, une poésie, une biodiversité complexe à sauvegarder.

\section{LE PROJET POUR DEMAIN : LA GESTION DURABLE}

La gestion forestière durable est aujourd'hui mondialement proclamée, notamment à la Conférence de Rio. Si j'ai bien compris la nuance, ce concept de durabilité viserait des objectifs plus généraux et une gestion plus globale de la forêt, associant ses diverses fonctions; ce concept exigerait une meilleure concertation entre les gestionnaires et tous les usagers de la forêt, une approche pluridisciplinaire et intersectorielle intégrant les autres activités environnantes, en particulier le développement rural, et notamment la production agricole. En effet, les pressions dommageables exercées sur les forêts sont souvent extérieures, notamment en zones tropicales et arides: une croissance démographique excessive, la pauvreté rurale, les besoins en terres (pour l'extension des terroirs agricoles), les besoins pastoraux et énergétiques que la forêt est souvent seule à pouvoir satisfaire.

Dans la mesure où cette gestion durable vise des objectifs plus ambitieux et élargis, les sylviculteurs ne peuvent qu'applaudir et adopter unanimement ce concept, tout en regrettant que la gestion à rendement soutenu n'ait pas été mise en œuvre plus tôt dans tous ces pays du monde où les forêts ont disparu. II y faut cependant mettre trois conditions :

- D'abord, il faut que les moyens humains, juridiques et financiers soient donnés. La gestion durable ne se décrète pas, elle est le long aboutissement d'un plan forestier dynamique et cohérent, porté par une réelle volonté politique, faisant converger ces moyens vers des gestionnaires compétents et responsables. Par ailleurs, il faudra bien décider qui va payer la mise en œuvre coûteuse de cette gestion, notamment les fonctions d'intérêt public revendiquées : l'État? les collectivités? le citoyen payeur de nouvelles taxes? C'est une question essentielle dont on parle trop peu.

- Ensuite, il faut que cette gestion durable obéisse à trois paramètres essentiels : intégrité écologique, équité sociale, rentabilitè. En particulier, elle ne doit pas négliger la fonction économique, non seulement utile à l'activité et à l'emploi, mais essentielle au plan cultural. 
- Enfin, il ne faudrait pas que cette gestion soit assujettie à des normes et des réglementations contraignantes, encadrée par des organismes internationaux lointains et budgétivores, décourageant les sylviculteurs responsables.

Concernant la première condition, avant que la gestion durable pénètre vraiment en forêt, une phase colossale d'élaboration de plans nationaux dégageant des moyens reste à faire en de nombreux pays où la forêt régresse. Les contraintes qui entravent aujourd'hui le développement durable en Afrique ont été bien analysées par Kone de la FAO. Avant de proclamer le développement durable, la vraie question est bien d'éliminer ces contraintes. Tel était l'objet des plans d'action forestiers initiés par la FAO. Le débat donne aujourd'hui l'impression qu'on peut se passer d'eux puisque le remède miracle du développement durable aurait été trouvé. Or c'est la stratégie contraire qu'il faut préconiser : faire d'abord les plans et ensuite promouvoir la gestion durable en application de ces plans.

Concernant la seconde condition, la crainte est bien réelle. À cette rencontre de mars 1995 évoquée plus haut, hors mes interventions non entendues, les mots économie, production, propriétaires forestiers, sylviculteurs, bois, n'ont jamais été prononcés en deux jours de débats ainsi que dans le communiqué final.

Sur le troisième point, le risque est également réel et nous y sommes d'ores et déjà confrontés, puisque la vague actuelle des débats internationaux en cours porte:

- d'une part, au plan technique, sur la définition de "critères" et d'“indicateurs" de gestion durable ;

— d'autre part, au plan commercial, sur l'invention d'un système d'écocertification qui garantirait aux consommateurs que les bois exploités proviennent d'une forêt bien gérée.

\section{L'ÉCOCERTIFICATION DES BOIS}

Le système est séduisant pour le consommateur constamment en quête de naturel en nos sociétés mal urbanisées, artificialisées, encombrées de nuisances. On lui donne la possibilité d'acheter un parquet ou une chaise avec un label tamponné comme une étiquette d'AOC sur une bouteille de vin, qui lui garantirait que la forêt d'où le bois provient fait l'objet d'une gestion durable contrôlée. Et en plus on donne à ce consommateur le sentiment qu'il participe à la grande œuvre de protection des patrimoines forestiers du monde dont on lui dit, par ailleurs, qu'ils sont tous et partout en grand danger de disparition.

Le discours serait merveilleux s'il ne cachait pas de redoutables effets pervers et parfois d'hypocrites intérêts commerciaux. Il pourrait d'ailleurs être généralisé à d'autres productions et d'autres échanges ; dans la même logique on pourrait imaginer, par exemple, un système de "socio-certification" à l'importation de produits fabriqués dans des conditions sociales détestables ; ainsi on pourrait penser à garantir que telle chemise n'a pas été fabriquée par des enfants d'Asie scandaleusement exploités.

Dans ce système de "socio-certification", il ne viendrait à l'idée de personne de le généraliser à tous les pays, y compris ceux qui disposent de bons salaires et d'une protection sociale avancée. Par contre, le système d'écocertification des bois" devrait être de portée universelle et s'appliquer à tous les pays, y compris ceux qui disposent depuis iongtemps de législations forestières éminemment protectrices et où des inventaires révèlent que le patrimoine forestier national s'enrichit et s'accroît.

Cette écocertification des bois a pu séduire quelques grands pays forestiers anglo-saxons du Nord, non pas par vertu forestière, mais plus prosaïquement parce qu'ils ont vite compris le parti commercial qu'ils pouvaient tirer d'un label avantageant leurs exportations de masse. En effet, ils ont des forêts de grande taille, homogènes, à dominante de résineux ; par contre, nos forêts plus morcelées, 
constituées d'essences plus diverses (ce qui est bon pour la biodiversité), de peuplements plus hétérogènes et variés (ce qui est bon pour le paysage et l'environnement) génèrent des lots de bois de petite taille dont le contrôle devient impossible, à moins de mettre en place des escouades de certificateurs qui dévoreraient le modeste revenu des sylviculteurs.

Par ailleurs, la mise en œuvre de cette "écocertification" paraît fort compliquée. En effet, l'arbre exploité s'éparpille en bois de feu, d'industrie et d'œuvre dont les destinations sont différentes. Le bois d'œuvre entrant en scierie en sort en sciages et en copeaux pour le feu et la trituration. Par ailleurs, la chaîne de transformation du bois, depuis son exploitation en forêt jusqu'à sa transformation industrielle, fait intervenir de nombreux acteurs: propriétaires, exploitants, débardeurs, transporteurs, première (scierie, tranchage) et seconde transformation. Et si une même chaise était faite avec des bois écocertifiés et d'autres qui ne le sont pas!

D'autre part, l'écocertification sera inévitablement coûteuse pour le sylviculteur car il est erroné de prétendre que le coût de l'écocertification sera supporté par le consommateur, le marché du bois étant mondialisé et fortement concurrentiel. Ainsi, le système est pervers pour l'ensemble de la filière car cet élément supplémentaire de coût de revient d'une production, dont la rentabilité est modeste, handicape le bois vis-à-vis d'autres matériaux qui le concurrencent.

Par ailleurs, la conception d'une stratégie commerciale sur une seule vertu écologique du bois écocertifié n'a aucun sens; elle peut tromper l'acheteur car le bois a d'autres qualités (technologiques, esthétiques, etc.) qui lui donnent sa vraie valeur. Le seul certificat de bonne conduite ne saurait attester la valeur d'un élève!

Mais il y a plus grave. S'il y a des bois qui sont écocertifiés, cela veut dire qu'il y en a d'autres qui ne le sont pas. La réussite du système veut que ces derniers soient boudès par le consommateur. Quel désastre pour ceux qui prônent le développement des emplois du bois! Quelle aubaine pour les matériaux concurrents qui peuvent être, par ailleurs, dévastateurs de l'environnement!

Quoi qu'il en soit, l'idée s'est déjà concrétisée dans quelques projets, faisant naître des vocations d'écocertificateurs. Les deux plus avancés paraissent être la procédure ISO (International Standard Organization) et le Système WWF-FSC (Forest Stewardship Council) qui ambitionne de devenir l'organisme accréditeur international sur la base de quelques grands principes. Mais là encore la théorie universelle se heurte aux réalités particulières. $\dot{A}$ titre d'exemple parmi bien d'autres, comment peut-on se rallier à ce principe : "Le FSC ne certifiera pas des plantations installees sur des terres occupées par des forêts naturelles ". II est la négation d'une politique de boisement et d'enrichissement qui peut créer de nouveaux peuplements incomparablement meilleurs que les anciens.

Mais la littérature touffue, que les débats internationaux produisent, commence de multiplier les contradictions et les interrogations. Une synthèse publièe dans Forêt-Entreprise évoque ce climat de doute qui apparaît : "En définitive, l'idée d'écocertification est plus intéressante dans les problèmes qu'elle révèle que dans les solutions qu'elle leur donne". Alors, de plus en plus, on semble se réfugier sagement sur un niveau national responsable (Wood Mark anglais) et rejeter l'écocertificateur international (ècolabel indonésien), etc. En effet, il serait aberrant que des critères et des labels en arrivent à compliquer des gestions satisfaisantes. On en arriverait à imposer aux bons élèves la discipline imaginée pour les mauvais.

La complexité, le coût, les effets pervers d'un tel système paraissent le condamner. Soyons néanmoins vigilants sur un sujet où la passion et l'utopie peuvent s'imposer au bon sens. Quoi qu'il en soit, s'il devait y avoir écocertification, elle devrait être de la seule responsabilité des États, en application du principe rappelé par la CNUED de "pleine souveraineté de ceux-ci". II y a deux raisons dèjà évoquées:

— parce que les situations forestières sont très différentes d'un pays à l'autre ; 
- parce que la gestion durable s'y réalise dans un cadre de mesures qui sont de la seule responsabilité des États.

Si la communauté internationale doit être investie d'une mission de supervision, il conviendrait :

- d'une part, d'en charger la FAO qui est la seule structure officielle du système des NationsUnies à disposer de compétences forestières dans son Département des Forêts;

- d'autre part, que cette supervision consiste non seulement à contrôler, mais surtout à promouvoir la gestion durable là où elle n'existe pas. À cet effet, les plans forestiers, notamment le tropical et le méditerranéen, injustement dénigrés par certains, devraient être fortement relancés.

\section{LES CRITÈRES ET LES INDICATEURS DE GESTION DURABLE}

Ils auraient pour objet de permettre aux écocertificateurs d'attester que les bois qu'ils labellisent proviennent effectivement de forêts durablement gérées. Des groupes de travail s'affairent sur ce sujet dans tous les coins du monde. Mais d'ores et déjà, il convient d'objecter les argumentations suivantes.

Une gestion durable qui serait contrôlée par des indicateurs ne se décrète pas, du jour au lendemain, comme une limitation de vitesse sur les routes. Elle est l'aboutissement d'une patiente et longue élaboration de politiques forestières nationales, mettant en place des structures adéquates administratives et professionnelles, un appareil de formation, de vulgarisation et de recherche, un dispositif législatif et réglementaire, des moyens humains, financiers, etc., etc. Tout cela demandera beaucoup de temps et incombe à chaque pays.

L'excellent document français "indicateurs de gestion durable" permet d'ores et déjà à notre pays d'attester que nos forêts sont durablement gérées. Citons quelques-uns de ces critères: le taux de boisement, la surface boisée rapportée au nombre d'habitants, les surfaces boisées enrichies, défrichées, chaque année, les surfaces des aires protégées (parcs, réserves), les moyens financiers affectés, etc., etc.

Le deuxième danger d'un tel dispositif est d'ouvrir la voie à des réglementations contraignantes mondialisées. Des pressions ont été exercées sur la Commission du Développement durable pour qu'elle se dote d'un "instrument de protection juridiquement contraignant", afin de lui permettre de passer des bons vœux actuels qu'elle répand à des directives obligatoires qu'elle pourrait imposer.

Mais surtout, si on pense qu'une harmonisation mondiale est souhaitable avant de s'engager dans la définition de ces critères internationaux, il faudrait déjà pouvoir imposer aux États deux priorités essentielles sans lesquelles toute prétention au développement global durable est illusoire.

D'abord mettre en place des systèmes d'observation des situations forestières actuelles et de leur évolution; or, de nombreux pays ont encore des statistiques forestières très incomplètes ou qui ne sont pas fiables. Chaque pays qui prétend au développement durable devrait disposer d'un instrument de mesure de l'état et de l'évolution de son patrimoine forestier, c'est-à-dire d'un inventaire forestier permanent, à cycle de l'ordre de la décennie.

Ensuite, au-delà des belles déclarations d'intentions, tout pays qui prétend au développement durable devrait disposer d'un plan d'action forestier national qui précise très concrètement l'organisation de ses institutions forestières, les actions prioritaires qu'il entend mener, les moyens financiers qu'il décide d'affecter, les outils juridiques dont il s'est doté, les personnels forestiers dont il dispose, etc., etc. Voilà ce qu'il faut surveiller!

Ce brouhaha international paraît inquiéter nos organisations professionnelles de la filière "ForêtBois". 


\section{J. GADANT}

M. Leclerc de Hauteclocque a proposé de faire reconnaître, au niveau international, l'excellence des gestions forestières pratiquées par certains pays, notamment la France. M. Jacques Delong, au nom des 11000 communes forestières de France, a interrogé le Ministre sur ce qu'il appelle la " farce de l'écocertification", risquant "de porter un préjudice considérable à la forêt et aux industries du bois". Enfin, le journal L'Officiel du Bois, organe des industries du bois, a livrè cette conclusion : "Ce mauvais dossier de l'écocertification des bois se pousse du col au niveau mondial. C'est un sujet sur lequel l'amont et l'aval de la filière doivent faire l'union sacrée" .

\section{ET POUR CONCLURE...}

II est excellent que la communauté internationale se rassemble sur ce thème de la forêt. Elle est investie d'une mission essentielle et clairement délimitée : promouvoir une fructueuse coopération internationale, susciter de réelles volontés politiques au niveau des États, mobiliser des financements plus copieux en faveur des plus pauvres. Quelle vaste tâche!

Dans la mosaïque extrêmement diversifiée des forêts du monde, ces velléités internationales à prétendre encadrer la gestion des forêts et la commercialisation des bois par des indicateurs ou des labels peuvent contribuer à alimenter de savants et tumultueux débats, mais resteront source de confusion et de complication inutile.

La stratégie à mener est, en définitive, fort simple ; elle se situe aux niveaux de deux acteurs :

- Sur le terrain : les gestionnaires des forêts, qu'elles soient publiques ou privées. II n'y a aucune raison de ne pas faire confiance à ces spécialistes dans la mesure où ils auront été correctement formés, où ils seront à l'écoute des usagers de la forêt et où ils disposeront de moyens.

- Au plan national, il appartient à l'État de définir une politique forestière cohérente et volontariste qui impose des obligations, dispense des moyens, contrôle l'excellence des gestions sur des bases scientifiques.

Entre l'international et le national, les rôles paraissent aussi clairement définis. Entre les écologistes intéressés par la forêt et les forestiers formés à l'écologie, le compagnonnage devrait être facile pour peu que chacun assume sa compétence.

La fable "Le vacher et le garde-chasse" du délicieux Florian de Sceaux exprimait cette antique sagesse. Le vacher s'était fait garde-chasse et le garde-chasse était devenu vacher. Résultat : le premier tue son chien à la place du chevreuil, le second s'endort dans la prairie et les vaches sont volées. Et le fabuliste de conclure :

"Que chacun fasse son métier

Et les vaches seront bien gardées".

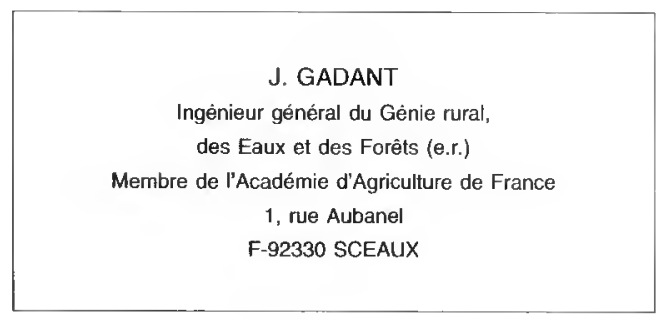




\section{QUAND L'ÉCOLOGIE DEVIENT NUISANCE (Résumé)}

Le vaste rassemblement de la Conférence des Nations-Unies sur l'Environnement et le Développement, tenue à Rio en 1992 , a mis la forêt en vedette et suscité des espoirs. Quel bitan en tirer? Sans doute une rareté de movens au regard d'une inflation de débats; mais aussi une localisation excessive sur le $\mathrm{E}$ d'environnement, minimisant le $\mathrm{D}$ du dèveloppement.

Ce texte demontre l'utopie d'un débat forestier mondialisè. II fait la chasse aux idées reçues et aux faux problèmes. II rappelle les contraintes pesant sur la forêt. II dènonce les mauvaises querelles et les accusations injustifiées faites aux gestionnaires des forêts.

L'auteur prône une gestion plurifonctionnelle équilibrée. II plaide pour le bois, matèriau le plus écologique qui soit. II proscrit toute contrainte bureaucratique imposée aux producteurs.

La promotion d'une bonne gestion, à la fois conservałrice du patrimoine et valorisatrice de toutes les fonctions, est la seule et vraie question. Plutôt que des contrôles a posteriori, la gestion durable exige, a priori, de la part des États, la délinition d'une politique forestière volontariste qui impose des obligations et dispense des moyens.

\section{WHEN ECOLOGY BECOMES NOXIOUS (Abstract)}

The massive gathering for the United Nations Conference on the Environment and Development held in Rio in 1992 brought forests into the limelight and raised hopes. What stands out today is a lack of resources alongside copious verbiage, but also an exaggerated focus on the $E$ of environment to the detriment of the $\mathbf{D}$ of development.

The paper shows how the idea of a worldwide debate on forests is utopian. It routs some common preconceptions and fallacies. It condemns the quibbling and false accusations brought against forest managers.

The author advocates a balanced multi-functional management method. He argues in favor of wood, the most ecological material there is, and against entorcing any form of bureaucratic constraint on producers.

Promoting proper management directed both at preserving the heritage and making full use of alt the potential is the only genuine option. Rather than calling on methods based on a posteriori inspections, sustainable management requires that States, a priori, define a concerted forest policy which both sets out obligations and allocates resources.

\section{WENN DIE ÖKOLOGIE SCHÄDLICH WÄRE (Zusammenfassung)}

Die grosse Zusammenkunft auf der Konferenz der Vereinten Nationen über Umwelt und Entwicklung, die 1992 in Rio stattfand, hat den Wald zum Mittelpunkt gemacht und Hoffnungen geweckt. Welche Bilanz kann man daraus ziehen ? Ohne Zweifel, ein Mangel an Mitteln gegenüber einer Diskussionsinflation; aber auch eine übertriebene Fokalisierung auf die Umwelt und eine Vemachlāssigung der Entwicklung.

Dieser Text zeigt die Utopie einer weltweiten Forstdebatte. Er macht Jagd auf vorgefaßte Meinungen und auf falsche Probleme. Er verweist auf die Zwangsmaßnahmen denen der Wald unterliegt. Er bringt die nichtigen Streitigkeiten und die ungerechten Anklagen denen die Fostverwalter ausgesetzt sind, an die Öffentlichkeit.

Der Verfasser setzt sich für einen ausgewogenen multifunktionellen Betrieb ein. Er plädiert für das Holz, das ökologischste Material das es gibt. Er weist jeden den Erzeugern auterlegten bürokratischen Zwang zurück.

Die Förderung einer guten Bewirtschaftung, zugleich Bewahrerin des Erbes und Aufwerterin aller Funktionen, ist die einzige whare Frage. Stat Kontrollen a posteriori, verlangt ein langfristiger Betrieb a prion, von seiten des Staates, die Formulierung einer voluntaristischen Forstpolitik, die Bedingungen auferlegt und Miftel bereitstellt.

\section{CUANDO LA ECOLOGIA SE VUELVE NOCIVA (Resumen)}

La grand reunión de la Conferencia de las Naciones Unidas, sobre el medio ambiente y el desarrollo, tenida en Rio de Janeiro en 1992 , ha puesto al bosque en candelero y ha suscitado esperanzas. ¿ Qué balance se puede deducir de ello ? Sin duda una pobreza de medios con respecto a la inflación de los debates; pero tambièn una focalización excesiva sobre la letra E del "entorno", minimizando la letra D del "desarrollo".

Dicho texto demuestra la utopía de un debate forestal mundializado. Evacúa las viejas ideas y los falsos problemas. Recuerda las obligaciones que pesan sobre el bosque. Denuncia las malas querellas y las acusaciones injustificadas contra los gestionarios del bosque.

El autor preconiza una gestión plurifuncional equilibrada. Aboga por la madera ; material ecológico por excelencia. Proscribe cualquier obligación burocràtica impuesta a los productores.

La promoción de una buena gestión, a la vez conservadora del patrimonio y valorizadora de todas las funciones : es la única y verdadera cuestión. Más bién que los controles a posteriori, de parte de los Estados, la definición de una politica forestal voluntarista que imponga obligaciones y lacilite los medios. 